\title{
A Case of UDP-Galactose 4'-Epimerase Deficiency Associated with Dyshematopoiesis and Atrioventricular Valve Malformations: An Exceptional Clinical Phenotype Explained by Altered N-Glycosylation with Relative Preservation of the Leloir Pathway
}

\begin{abstract}
Christopher A. Febres-Aldana $^{a} \quad$ Liset Pelaez $^{\text {b, c }}$ Meredith S. Wright ${ }^{d}$ Ossama M. Maher ${ }^{e}$ Anthony J. Febres-Aldana ${ }^{f}$ Jun Sasakic,g Parul Jayakar ${ }^{\text {h, }}$ Anuj Jayakar $^{\mathrm{i}}$ Magaly DiazBarbosa $^{i}$ Michelin Janvier ${ }^{i}$ Bala Totapally ${ }^{c, i, j}$ Daria Salyakina ${ }^{i} \quad$ Jorge R. Galvez-Silva ${ }^{e, k}$

${ }^{a} A M$ Rywlin, MD, Department of Pathology and Laboratory Medicine, Mount Sinai Medical Center, Miami Beach, FL, USA; 'Department of Pathology and Clinical Laboratories, Nicklaus Children's Hospital, Miami, FL, USA; 'Herbert Wertheim College of Medicine, Florida International University, Miami, FL, USA; Rady Children's Institute for Genomic Medicine, San Diego, CA, USA; 'Department of Pediatric Hematology/Oncology, Nicklaus Children's Hospital, Miami, FL, USA; fDepartment of Internal Medicine, Mount Sinai Medical Center, Miami Beach, FL, USA; gDepartment of Cardiology, Nicklaus Children's Hospital, Miami, FL, USA; hivision of Genetics and Metabolism, Nicklaus Children's Hospital, Miami, FL, USA; 'Personalized Medicine Initiative, Nicklaus Children's Hospital, Miami, FL, USA; 'Division of Critical Care Medicine, Nicklaus Children's Hospital, Miami, FL, USA; ${ }^{k}$ Bone Marrow Transplant Program, Nicklaus Children's Hospital, Miami, FL, USA
\end{abstract}

\section{Established Facts}

- The generalized form of UDP-galactose- $4^{\prime}$-epimerase (GALE) deficiency manifests with signs and symptoms of galactosemia including seizures, cognitive impairment, hypotonia, failure to thrive, liver failure, aminoaciduria, and cataracts.

- Individuals with non-generalized forms of GALE deficiency may remain asymptomatic and undiagnosed with uncertain outcomes.

- The missense GALE mutation p.R51W has been associated with inherited thrombocytopenia.

\section{Novel Insights}

- GALE deficiency is associated with abnormal heart valve development and bone marrow dysfunction in the absence of classic galactosemia manifestations.

- The compound heterozygous p.R51W/p.G237D of GALE is pathogenic.

- Abnormal $N$-glycosylation may explain the phenotypic manifestations of non-generalized forms of GALE deficiency.

Christopher A.Febres-Aldana

AM Rywlin MD, Department of Pathology and Laboratory Medicine Mount Sinai Medical Center

4300 Alton Road, Suite 2400, Miami Beach, FL 33140 (USA)

chrisfeb.2009@gmail.com 


\section{Keywords}

Galactosemia · GALE deficiency · Glycosylation · Bone marrow failure disorders $\cdot$ Congenital heart defects

\section{Abstract}

The generalized form of UDP-galactose-4'-epimerase (GALE) deficiency causes hypotonia, failure to thrive, cataracts, and liver failure. Individuals with non-generalized forms may remain asymptomatic with uncertain long-term outcomes. We report a 2-year-old child compound heterozygous for GALE p.R51W/p.G237D who never developed symptoms of classic galactosemia but has a history of congenital combined mitral and tricuspid valve malformation and pyloric stenosis, and presented with pancytopenia. Variant pathogenicity was supported by predictive computational tools and decreased GALE activity measured in erythrocytes. GALE function extends to the biosynthesis of glycans by epimerization of UDP- $\mathrm{N}$-acetyl-galactosamine and -glucosamine. Interrogation of the Gene Ontology consortium database revealed several putative proteins involved in normal hematopoiesis and atrioventricular valve morphogenesis, requiring $\mathrm{N}$-glycosylation for adequate functionality. We hypothesize that by limiting substrate supply due to GALE deficiency, alterations in $\mathrm{N}$-linked protein glycosylation can explain the patient's phenotype.

(c) 2020 S. Karger AG, Basel

\section{Introduction}

Galactosemia is the term used to describe inborn errors of the metabolism characterized by the accumulation of galactose due to mutations in enzymes of the Leloir pathway, including galactose (gal)-1-phosphate uridylyltransferase (GALT), galactokinase, and UDP-gal-4' epimerase (GALE) [Holden et al., 2003; Fridovich-Keil et al., 2016]. GALE deficiency (OMIM 230350) is inherited in an autosomal recessive manner with an estimated prevalence of $1 / 7,000$ in African-American and about 1/70,000 among American infants of European ancestry [Pyhtila et al., 2015]. The clinical manifestations correlate with accumulated total galactose (gal and gal-1-phosphate) [Openo et al., 2006; Fridovich-Keil et al., 2016]. Epimerase deficiency galactosemia can be divided by apparent enzyme activity levels into the following 3 forms: generalized, peripheral, and intermediate [Fridovich-Keil et al., 2016].

Generalized GALE deficiency is mainly observed in patients homozygous for the variant p.V94M [c.280G>A].
These individuals develop neonatal galactosemia characterized by hypotonia, failure to thrive, vomiting, weight loss, aminoaciduria, cataracts, jaundice, hepatomegaly, and liver dysfunction [Dias Costa et al., 2017]. Cardiac failure and dysmorphic features, including micrognathia, finger deformities, bilateral hip dysplasia, and talipes equinovarus, are among other early noticeable but less common manifestations [Dias Costa et al., 2017]. Some patients develop cognitive-developmental delay and sensorineural loss regardless of dietary restrictions [Fridovich-Keil et al., 2016]. Neonates with the intermediate or peripheral forms are usually asymptomatic even on a regular milk diet and are only identified following biochemical detection of elevated total galactose on newborn screening. Patients with non-generalized forms of GALE deficiency have been treated by dietary galactose/lactose restriction, at least in infancy, and thus far, those who have been followed appear to remain clinically well [Fridovich-Keil et al., 2016]. Still, the long-term outcome remains unclear.

The epimerase activity of GALE extends outside the Leloir pathway to the generation of substrates for the biosynthesis of glycoproteins and glycolipids by the interconversion of UDP- $N$-acetyl-galactosamine (UDPgalNAc) and UDP- $N$-acetyl-glucosamine (UDPglcNAc) [Holden et al., 2003; Broussard et al., 2020]. Failure to produce sufficient UDP-gal, UDP-glc, UDPgalNAc, and UDP-glcNAc substrates can alter numerous biological processes regulated by $\mathrm{N}$-linked and $\mathrm{O}$ linked glycosylation [Broussard et al., 2020]. Proper glycosylation is critical to normal hematopoiesis, in particular to megakaryocyte and platelet development, as reflected in the presence of thrombocytopenia in the context of congenital disorders of glycosylation [Seo et al., 2019]. Although pathogenic variants in GALE have not previously been associated with thrombocytopenia, Seo et al. [2019] suggested that GALE mutation is inadequate for normal glycosylation and thereby may impair megakaryocyte and platelet development. Individuals with inherited thrombocytopenia harboring the GALE mutation p.R51W can also develop mild anemia and febrile neutropenia in the absence of classic symptoms of galactosemia [Seo et al., 2019]. The consequences of GALE deficiency are yet to be fully defined, and some manifestations probably result from disruption of protein glycosylation [Broussard et al., 2020]. In this report, we discuss how abnormalities in $\mathrm{N}$-glycosylation can explain the congenital heart disease and bone marrow dysfunction of a child with a non-generalized form of GALE deficiency. 


\section{Case Presentation}

A Hispanic 2-year-old boy was admitted because of fever and pancytopenia in the setting of a respiratory syncytial virus infection. His history was remarkable for severe regurgitation of both atrioventricular valves (Fig. 1) and pyloric stenosis. At 5 weeks of age, he underwent mechanical valve replacement of the mitral valve, tricuspid valvuloplasty, and pyloromyotomy. The preoperative echocardiogram had shown redundant mitral and tricuspid valve leaflets resulting in poor coaptation and a small ostium secundum-type atrial septal defect. The postoperative period was complicated by a nosocomial infection and pancytopenia. The patient remained pancytopenic until he was 4 months old, when the blood cell counts started to increase reaching a full recovery at 8 months of age. The blood cell counts remained within normal limits until the current admission. Routine oral medications included warfarin and aspirin. He was on a regular diet including fruits, vegetables, and up to $32 \mathrm{oz}$. of regular milk per day. The patient was placed on a lactose-free diet upon diagnosis.

On admission, the patient was febrile $\left(100.6^{\circ} \mathrm{F}\right)$ with tachycardia $(145 \mathrm{bpm})$. His weight $(15.8 \mathrm{~kg})$ and height $(92 \mathrm{~cm})$ were at the 90th percentile for age. Laboratory tests revealed a white blood cell (WBC) count of $1.9 \times 10^{9} / \mathrm{L}$ (range of reference [RR]: 4.8-10.8), absolute neutrophil count (ANC) of 864 cells/ $\mu \mathrm{L}$ (RR: $1,000-$ $6,000)$, hemoglobin (Hb) of $9.2 \mathrm{~g} / \mathrm{dL}$ (RR: 10.5-14.8), hematocrit of $27.9 \%$ (RR: $31-41$ ), and platelet count of $13.9 \times 10^{9} / \mathrm{L}$ (RR: 150 500). Acute-phase reactants, including procalcitonin, were elevated. Coagulation tests were abnormal consistent with warfarin effect. Additional tests, including electrolytes, renal function markers, liver enzymes, total bilirubin, $\mathrm{LDH}$, vitamin $\mathrm{B} 12$, folate, and urinalysis, were within normal limits. Imaging studies showed findings consistent with left lower lobe pneumonia, right orbital cellulitis, and mild splenomegaly $(9.7 \mathrm{~cm})$. Blood cultures were negative, but next-generation sequencing for microbial cell-free DNA detected Streptococcus pneumoniae, Fusobacterium necrophorum, and Bacteroides vulgatus. The initial management was broad-spectrum antibiotic therapy and multiple transfusion of blood products, including 6 units of platelet and 3 units of packed red blood cell. The patient's fever improved but he continued to be pancytopenic.

\section{Methods and Results}

\section{Bone Marrow Biopsy}

A bone marrow biopsy performed on the fourth hospitalization day showed an increased myelomonocytic population (monocytes: $24 \%$ ) with immature features and cytoplasmic vacuoles containing engulfed red blood cells (RBCs) and cellular debris (Fig. 2, whole slide images available at https://kikoxp.com/posts/2283). Erythroid precursors exhibited nuclear irregularities and megaloblastic changes in more than $10 \%$. Few neutrophils were hypogranular and hypersegmented. There was megakaryocytic hyperplasia with few small hypolobated forms. A reticulin stain revealed minimal fibrosis. The karyotype, flow cytometry, and FISH panels for myelodysplastic syndrome and acute leukemia were unremarkable.

Increased ferritin $(219 \mu \mathrm{g} / \mathrm{L}, \mathrm{RR}: 8-77)$, increased triacylglyceride (252 mg/dL, RR: 27-125), cytopenias, splenomegaly, fever and
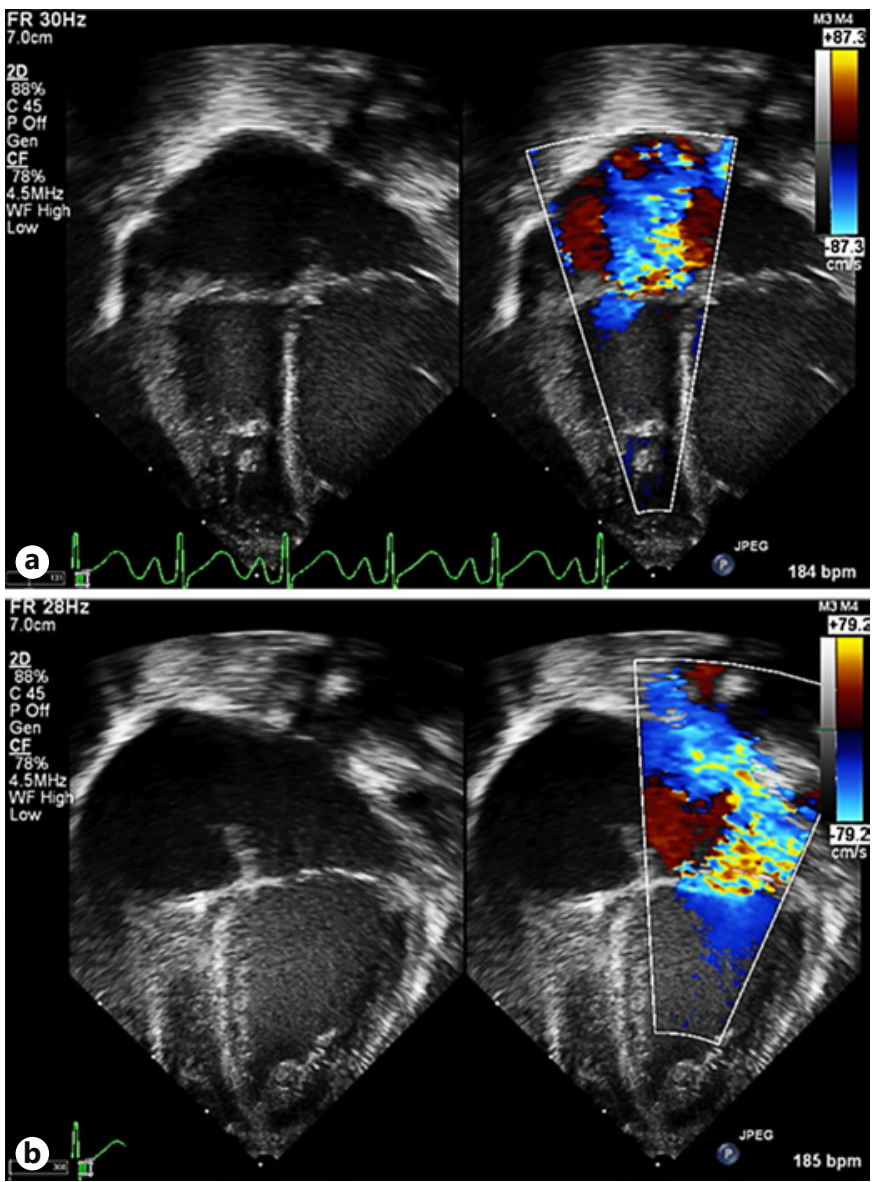

Fig. 1. Neonatal echocardiographic color images in systole showing severe tricuspid valve regurgitation (a) and severe mitral valve regurgitation $(\mathbf{b})$.

hemophagocytosis were suggestive of hemophagocytic lymphohistiocytosis. However, soluble interleukin-2 receptor was normal (929 U/mL, RR: 398-1,940), fibrinogen (609, RR: 200-400) was elevated, and flow cytometry granzyme/perforin assay for natural killer cell abnormalities was normal. Parvovirus, cytomegalovirus and Epstein-Barr virus infections were ruled out. Filgrastim (70 $\mu \mathrm{g} /$ day $)$, dexamethasone $(2.5 \mathrm{mg} / 8 \mathrm{~h})$, and 2 doses of intravenous immunoglobulin were administered, leading to partial improvement of WBC and platelet counts.

After 4 weeks of hospitalization, the patient's overall condition improved (Hb: $8.4 \mathrm{~g} / \mathrm{dL}, \mathrm{WBC}: 2.3 \times 10^{9} / \mathrm{L}$, Platelets: $162 \times 10^{9} / \mathrm{L}$ ). A follow-up bone marrow biopsy showed regenerative features, erythroid hyperplasia with no atypia and normal megakaryopoiesis. The numbers of monocytes decreased (5\%) with no evidence of hemophagocytosis or vacuolization. During the 8 months after being discharged home, the patient has been asymptomatic and in stable conditions; however, the pancytopenia became a chronic condition(range of values registered in the follow-up period: WBC: $2-4 \times 10^{9} / \mathrm{L}$, ANC: $1,000-1,500$ cells $/ \mu \mathrm{L}, \mathrm{Hb}: 6-9 \mathrm{~g} / \mathrm{dL}$, Platelets: $\left.40-120 \times 10^{9} / \mathrm{L}\right)$. 
Fig. 2. Bone marrow findings on fourth hospitalization day. a Core biopsy showing cellular marrow with megakaryocytic hyperplasia and hypolobated forms (arrowhead), $\times 400$. $\mathbf{b}$ Bone marrow aspirate with atypical myelomonocytic precursors containing cytoplasmic vacuoles (arrowheads), binucleated forms (continuous arrow), and atypical erythroid precursors (dashed arrows), ×600. c, d Erythrophagocytosis in a promonocyte (c), a myeloid precursor $(\mathbf{d}), \times 1000$. e Hypersegmented neutrophil with cytoplasmic vacuoles, $\times 1000$. All microphotographs correspond to Wright-Giemsa staining. Whole slide images available at https://kikoxp.com/ posts/2283.

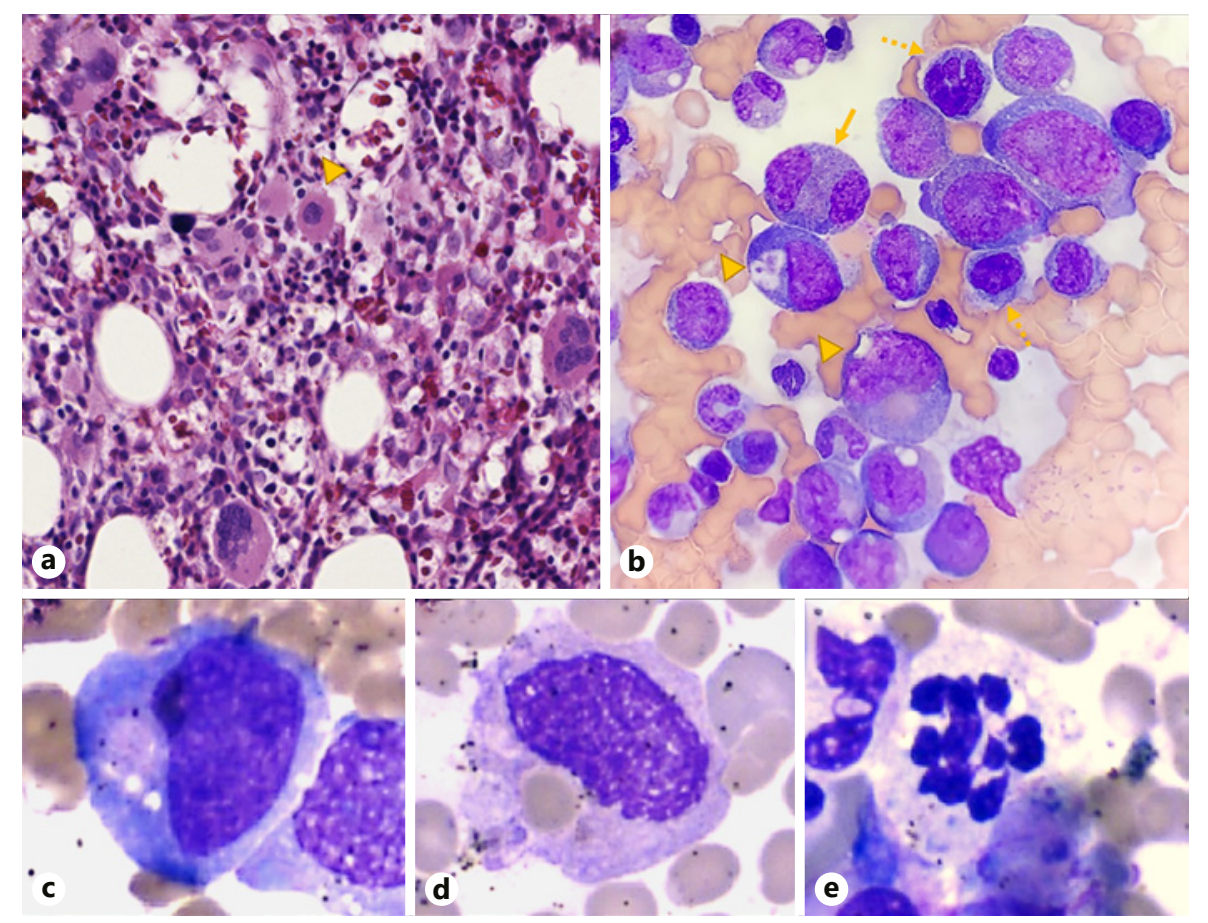

\section{Whole-Genome Sequencing}

Blood was drawn following parental consent for whole-genome sequencing (WGS) including a targeted variant sequencing of parental samples for inheritance determination. DNA was extracted and sequenced on a NovaSeq (Illumina, San Diego, CA, USA) [S2 flow cell]. Rapid read alignment and nucleotide variant calling was performed using the Edico Dragen processor (Illumina). Yield was $127.5 \mathrm{~Gb}$ for the proband resulting in 4,916,104 distinct variant calls. Variant analysis and interpretation were performed using Fabric Enterprise platform (Fabric Genomics), which employs proprietary algorithms to prioritize candidate variants by integrating phenotypic and genomic data using the following Human Phenotype Ontology (HPO) terms [Köhler et al., 2017]: Mitral regurgitation (HP:0001653), thrombocytopenia (HP:0001873), pancytopenia (HP:0001876), leukopenia (HP:0001882), and recurrent infections (HP:0002719). Variants were filtered to retain those with allele frequencies of $<0.5 \%$ in multiple population databases including gnom $A D$, Exome Variant Server, 1000 Genomes, and ExAC. A custom gene panel containing 1,261 genes was then created using Phenolyzer [Yang et al., 2015] with the above HPO terms.

\section{Variant Interpretation}

This method identified 2 missense changes heterozygous in the GALE gene: a maternally inherited likely pathogenic heterozygous c.151C>T (p.R51W) variant and a paternally inherited heterozygous c.710G $>$ A (p.G237D) variant of uncertain significance. The p. R51W variant was previously reported as a homozygous change in multiple affected individuals from the same kindred with histories of thrombocytopenia, intracranial bleeding, anemia, and febrile neutropenia [Seo et al., 2019]. The variant segregated with disease in this kindred. Functional characterization of GALE
p.R51W demonstrated significantly lower enzymatic activity and greatly increased thermal instability [Seo et al., 2019]. It is present in the heterozygous state in the gnomAD v2.1.1 population database at a frequency of $0.0000199(5 / 250,730)$ and thus is rare. Based on the available evidence, c.151C $>\mathrm{T}$ (p.R51W) is classified as likely pathogenic by the American College of Medical Genetics (ACMG) scoring guidelines [Richards et al., 2015].

The p.G237D variant has not been previously reported or functionally characterized in the literature to our knowledge. It is present in the heterozygous state at a frequency of 0.00000821 $(2 / 243,570)$, being reported in a Latino population only. ClinVar contains an entry for this variant (Variation ID: 596688) with one classification as of uncertain significance. Predictive in silico tools support p.G237D to have a deleterious effect on protein function (Fig. 3; Table 1). MutPredV2 revealed a high pathogenicity score for p.G237D causing an alteration of the catalytic site and binding substrate interface. The residue 237 has a high conservation score by the Genomic Evolutionary Rate Profiling (GERP) tool comparing 35 mammal orthologues and Molecular Evolutionary Genetic Analysis (MEGA) X comparing 94 taxa including prokaryotes. Based on the available evidence, the c.710G >A (p.G237D) variant was classified according to ACMG guidelines as a variant of uncertain significance.

\section{Biochemistry}

Levels of GALT activity were normal. The level of gal-1-phosphate was increased in RBCs $14.1 \mathrm{mg} / \mathrm{dL}$ (normal: $\leq 0.9$ ). Urine level of galactitol was normal. The GALE activity in RBCs, assessed by liquid chromatography with tandem mass spectrometry, was low $0.9 \mathrm{nmol} / \mathrm{h} / \mathrm{mg} \mathrm{Hb}$, (normal: $\geq 3.5$ ) supporting the diagnosis of GALE deficiency.
GALE Deficiency Associated with Dyshematopoiesis and Congenital Heart Disease
Mol Syndromol 2020;11:320-329

DOI: $10.1159 / 000511343$ 


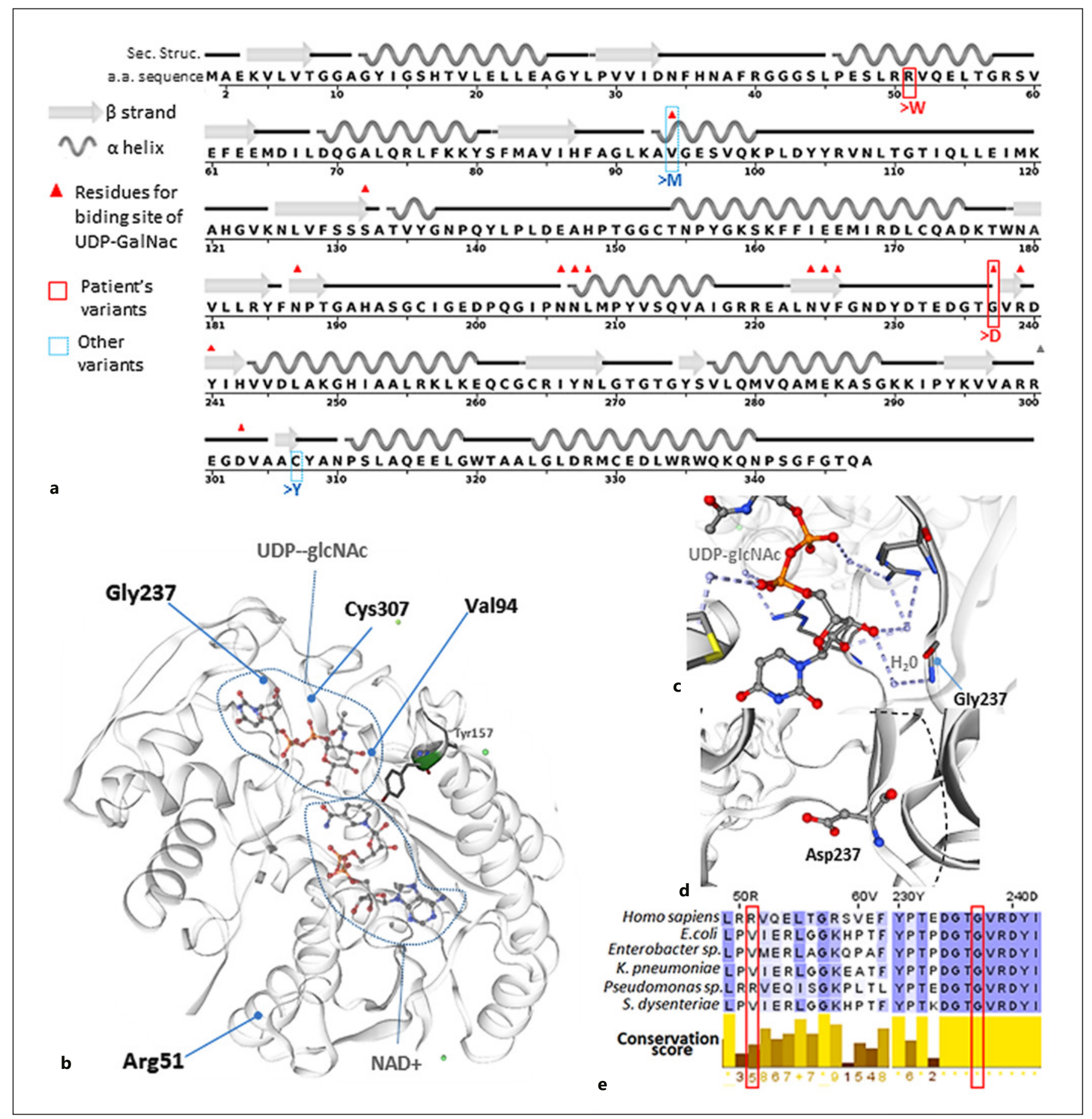

Fig. 3. Representation of the primary and secondary protein structure of GALE with the variants p.R51W, p.G237D. a Amino acid (a.a.) sequence and predicted secondary structure (arrow: $\beta$-strand, wavy line: $\alpha$-helix, and flat line: random conformation) with active site residues that bind UDP-galNAc (red triangle). Modeled at RCSB Protein Data Bank (PDB ID: 1HZJ and 1XEL). In this example, changes in residues 94 and 237 are predicted to alter the binding site for GALE substrates. $\mathbf{b}$ Overall 3D protein structure of GALE wild type generated by the SWISS-MODEL homology-modelling pipe- line server (template ID: Q14376). The residues valine 94, glycine 237, and cysteine 307 delimit the substrate binding pocket. Changes in these amino acids may modify the substrate specificity and affinity. Arginine 51 is located in an a-helix domain away from the catalytic site. c In GALE wild type, glycine 237 forms a water bridge with UDP-glcNAc (atom: O3C). d Aspartate 237 adds a negative charge and larger side chain causing steric interference to substrate binding. e Evolutionary analysis using MEGA X. High conservation scores for residue 237, while for residue 51 the conservation is lower. 
Table 1. Pathogenicity of the patient's GALE variants p.G237D and p.R51W, and p.V94M and p.C307Y for comparison

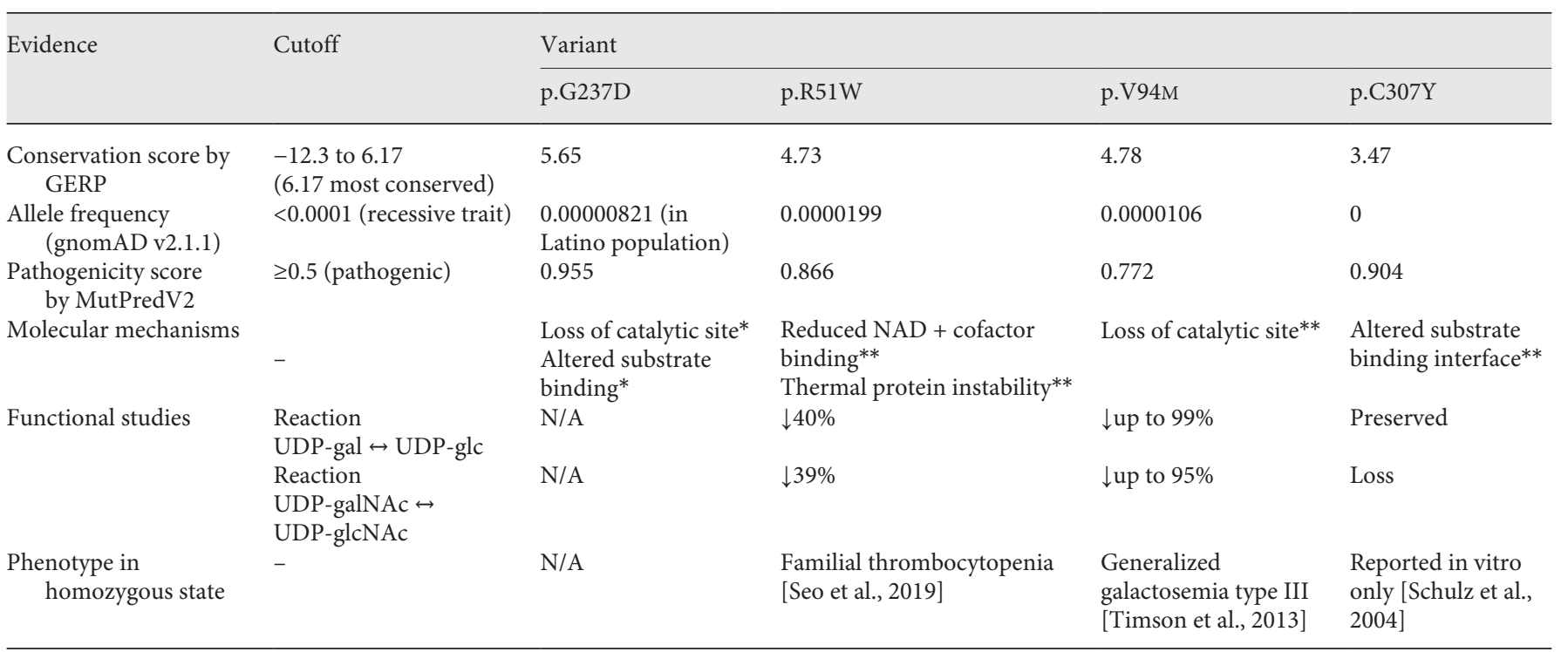

N/A, not available. ${ }^{*}$ By MutPredV2 in silico analysis; ${ }^{* *}$ confirmed in vitro.

\section{Discussion}

This report highlights some fundamental concepts related to the factors shaping the clinical expression of GALE genotypes as well as the importance of $N$-linked protein glycosylation in ensuring a normal hematopoiesis and organ morphogenesis. The patient presented with congenital combined mitral and tricuspid malformation, pyloric stenosis, and infection-induced episodes of pancytopenia in the absence of classic signs of galactosemia despite having genetic and biochemical evidence of GALE deficiency.

The missense GALE mutations p.S81R, p.T150M, p.K161N, p.D175N, p.K257R, p.P293L, and p.G319E are commonly found in compound heterozygotes of nonconsanguineous origin, usually exhibit partial impairment of GALE activity (15-64\%), and are associated with non-generalized forms or asymptomatic presentations [Wasilenko et al., 2005; Openo et al., 2006; McCorvie et al., 2012]. In these individuals, the emergence of galactosemia symptoms depends on the amount and duration of exposure to gal. Therefore, moderate and mild epimerase deficiency galactosemia is likely to remain unrecognized. This problem is aggravated by the fact that the preferred method of newborn screening for galactosemia, in many US states including Florida, is a fluorescence assay that measures GALT activity but not GALE activity [DeClue et al., 1991]. The diagnosis of GALE deficiency requires the demonstration of decreased GALE activity and the identification of biallelic pathogenic variants. Molecular testing for galactosemia is clinically biased to patients with classic symptoms; in consequence, the outcomes associated with intermediate GALE deficiency are unknown and likely misdiagnosed. In our case, a phenotype-driven WGS panel allowed the fortuitous detection of GALE p.R51W/p.G237D, which correlated with a decrease in GALE activity. From a clinical standpoint, the challenge is to decide to what extent the patient's signs and symptoms can be attributed to GALE deficiency.

The pathogenicity analysis of the patient's GALE variants was complicated by the absence of prior functional and clinical data. Predictive modeling tools suggest that p.G237D is as deleterious as p.V94M, and the residual patient's GALE activity is possibly the result of p.R51Wderived polypeptides only. The GALE activity recorded in p.R51W homozygotes was previously estimated to be around $60 \%$ of wild type [Seo et al., 2019]. Notwithstanding, considering that GALE is found as homodimers with inter-subunit interactions [Timson and Lindert, 2013], a substantial proportion of the patient's GALE activity is probably derived from compound p.G237D/p.R51W homodimers. In this situation, enzymatic kinetic analysis is useful to ascertain the degree of activity impairment.

Complete loss of GALE activity has been reported in vitro only and is probably incompatible with life. Homozygotes for p.V94M, which is the most severe tolerated 


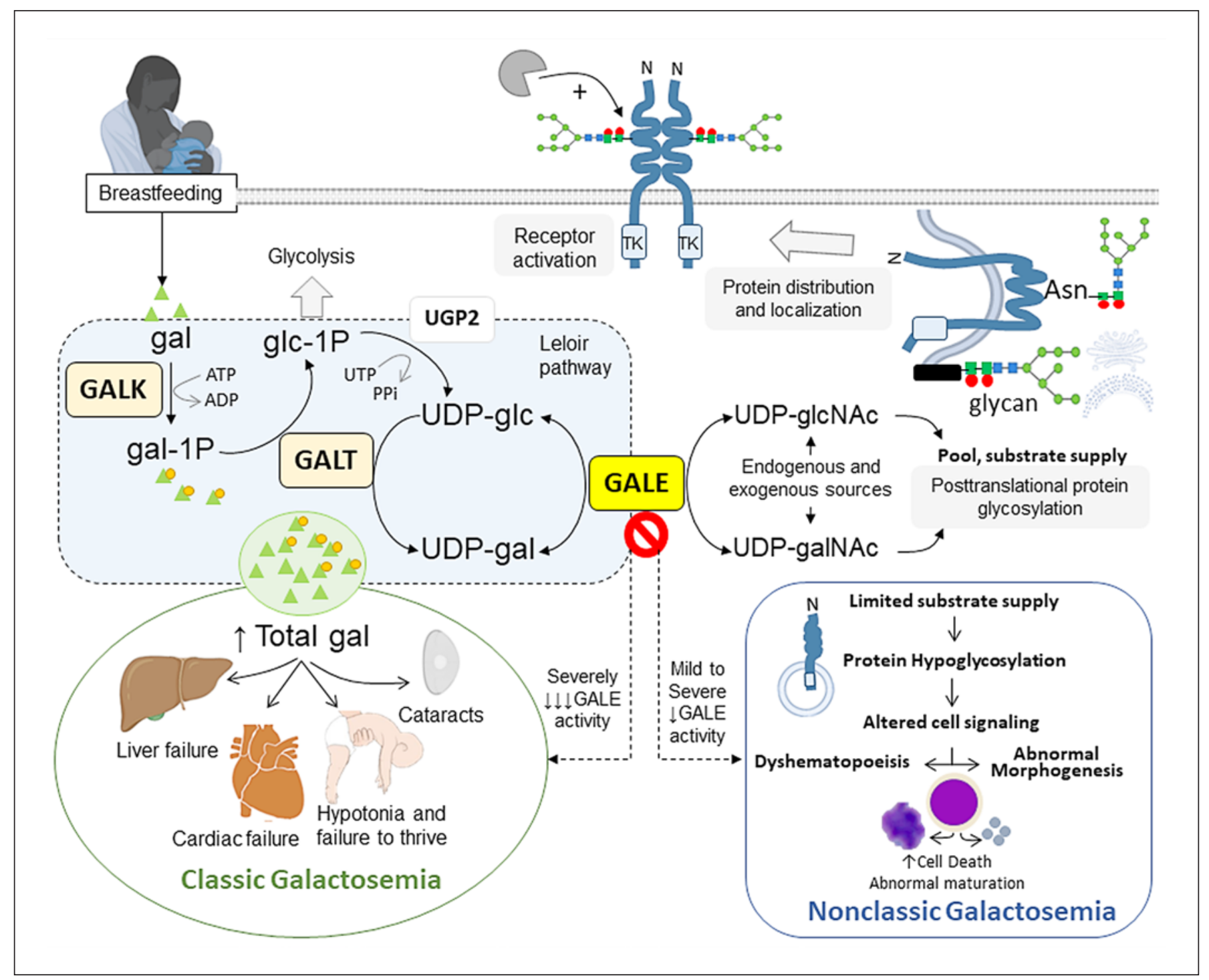

Fig. 4. Potential mechanisms of disease in GALE deficiency. UDPgalactose- $4^{\prime}$-epimerase (GALE) along with galactokinase 1 (GALK) and galactose-1-phosphate uridylyltransferase (GALT) constitute the Leloir pathway for the catabolism of galactose. Individuals with generalized GALE deficiency will develop symptoms of galactosemia, liver and cardiac failure, hypotonia, failure to thrive and cataracts, after breastfeeding or milk diet in association with an increase in total galactose. Intermediate forms of GALE deficiency may potentially lead to developmental and hematologic disorders by disturbing posttranslational protein glycosylation.

genotype, still display at least $5 \%$ of residual epimerase activity for UDP-galNAc and 1\% for UDP-gal [Wohlers et al., 1999]. The activity reduction can also vary according to the substrate and cell type evaluated [Openo et al., 2006]. Although it is unknown which loss of activity has a greater impact in defining GALE-deficient patients'
Endogenous and exogenous sources of UDP-glcNAc and UDPgalNAc may not compensate the substrate pool supply for glycan biosynthesis during increased demand. $\mathrm{N}$-glycosylation regulates the distribution, localization, and activation of many proteins including receptors with tyrosine kinase activity. States of cell surface hypoglycosylation are associated with a decrease in survival signals and increased susceptibility to cell death. Abnormal protein glycosylation may explain the manifestations, other than classic galactosemia, observed in individuals with GALE deficiency.

phenotype, the enzymatic activity observed in non-generalized forms may be "sufficient" to halt an excessive accumulation of total gal, making acute galactosemia less likely to develop. This residual activity may be "insufficient" to compensate for an increase in the demand of UDP-glcNAc/UDP-galNAc substrates in cells where the 
Table 2. Sites of $N$-Linked glycosylation, predicted by asparagine residues, in proteins involved in atrioventricular valve morphogenesis (GO: 0003181), foregut morphogenesis (GO: 0007440), and hematopoiesis (GO: 0060216, 0030219, and 0061515) obtained at the GO consortium resource database and UniProt Knowledgebase

\begin{tabular}{|c|c|c|c|c|c|}
\hline Gene & Protein & Asparagine position & Topological domain & Biological process & Role in development \\
\hline$A R V C 1$ & Activin A receptor, type 1 & 102 & Extracellular & \multirow[t]{4}{*}{ AV valve morphogenesi } & isRole in early phases of endocardial cushion formation \\
\hline$B M P 2$ & Bone morphogenetic protein 2 & $87,135,163,164,200,338$ & Propeptide, chain & & Coordination of AV canal formation \\
\hline$B M P R 1 A$ & $\begin{array}{l}\text { Bone morphogenetic protein receptor, } \\
\text { type 1A (aka ALK3) }\end{array}$ & 73 & Extracellular & & $\begin{array}{l}\text { Determination of length and position of leaflet, and annulus } \\
\text { fibrous formation }\end{array}$ \\
\hline BMPR2 & $\begin{array}{l}\text { Bone morphogenetic protein receptor, } \\
\text { type } 2\end{array}$ & $55,110,126$ & Chain & & \\
\hline CSF1 & Macrophage colony-stimulating factor 1 & $154,172,363,365$ & Lumenal & \multirow[t]{2}{*}{ Hematopoiesis } & \multirow[t]{2}{*}{$\begin{array}{l}\text { Regulation of survival, proliferation and differentiation of } \\
\text { mononuclear phagocytes }\end{array}$} \\
\hline CSF2 & $\begin{array}{l}\text { Granulocyte-macrophage colony- } \\
\text { stimulating factor }\end{array}$ & $22,24,26,27,44,54$ & Chain & & \\
\hline DCHS1 & Protocadherin-16 & $\begin{array}{l}217,256,402,584,1,249,1,521,1718 \\
1,996,2,361,2,428,2,569,2,761,2,792 \\
2,862\end{array}$ & Chain & $\begin{array}{l}\text { Mitral valve leaflet } \\
\text { formation }\end{array}$ & $\begin{array}{l}\text { Determination of valve size and shape by influencing cellular } \\
\text { migration and patterning } \\
\text { Variants associated with mitral valve prolapse with } \\
\text { abnormally elongated and thickened leaflets }\end{array}$ \\
\hline EFNA1 & Ephrin A1 & 26 & Chain & $\begin{array}{l}\text { Mitral valve } \\
\text { morphogenesis }\end{array}$ & Regulates cellularity in endocardial cushions \\
\hline FGF2 & Fibroblast growth factor receptor 2 & $83,123,228,241,265,297,318,331$ & Extracellular & \multirow[t]{4}{*}{ Hematopoiesis } & \multirow{2}{*}{$\begin{array}{l}\text { Antiapoptotic effect in hematopoietic precursors } \\
\text { Location and receptor autoactivation is dependent on } \\
\text { glycosylation }\end{array}$} \\
\hline FGF3 & Fibroblast growth factor receptor 3 & $98,225,262,294,315,328$ & Extracellular & & \\
\hline FLT3 & Fms-like tyrosine kinase 3 & \multicolumn{2}{|c|}{$\begin{array}{l}43,100,151,306,323,351,354,473,502 \text {, Extracellular } \\
541\end{array}$} & & $\begin{array}{l}\text { Induces differentiation, proliferation and survival of } \\
\text { hematopoietic progenitor cells } \\
\text { Decreased } N \text {-glycosylation attenuated FLT3 signaling }\end{array}$ \\
\hline IL3RA & Interleukin-3 receptor subunit alpha & $46,64,80,109,212,218$ & Extracellular & & Monocyte differentiation \\
\hline KIT & Stem cell growth factor receptor kit & $\begin{array}{l}130,145,283,293,300,320,352,367 \\
463,486\end{array}$ & Extracellular & \multirow[t]{2}{*}{ Hematopoiesis } & $\begin{array}{l}\text { Induces differentiation, proliferation and survival of } \\
\text { hematopoietic stem/progenitor cells }\end{array}$ \\
\hline$M P L$ & Thrombopoeitin receptor & $117,178,298,358$ & Extracellular & & Positive regulator of megakaryocyte development \\
\hline NOTCH1 & $\begin{array}{l}\text { Neurogenic locus notch homolog } \\
\text { protein } 1\end{array}$ & $41,959,1,179,1,241,1,489,1587$ & Extracellular & $\begin{array}{l}\text { AV valve and foregut } \\
\text { morphogenesis }\end{array}$ & $\begin{array}{l}\text { Regulates cellularity in cardiac valvular primordia by } \\
\text { modulation of EMT }\end{array}$ \\
\hline SLIT3 & Slit homolog 3 protein & $\begin{array}{l}72,192,563,622,784,792,797,928 \\
1,008,1,025,1,181,1,247,1,406\end{array}$ & Chain & \multirow{3}{*}{ AV valve morphogenesi } & $\begin{array}{l}\text { isRegulates cellularity in cardiac valvular primordia by } \\
\text { modulation of Notch signaling/EMT }\end{array}$ \\
\hline TGFB2 & Transforming growth factor beta- 2 & $72,140,241$ & Chain & & Ventricular septation \\
\hline TGFBR2 & TGF-beta receptor type- 2 & $70,94,154$ & Chain & & \\
\hline THPO & Thrombopoietin & $197,206,235,249,256,336,351$ & Chain & Hematopoiesis & Positive regulator of megakaryocyte development \\
\hline
\end{tabular}

AV, atrioventricular; EMT, epithelial-to-mesenchymal transition.

degree of protein and lipid glycosylation influences the activity of canonical processes such as cellular survival, differentiation and regulated death (Fig. 4).

The Gene Ontology (GO) consortium resource database was interrogated to identify proteins undergoing $\mathrm{N}$ glycosylation involved in atrioventricular valve morphogenesis, foregut morphogenesis, and hematopoiesis, providing a better insight on how the p.R51W/p.G237D genotype resulted in the patient's bone marrow dysfunction and congenital abnormalities (Table 2). Putative targets included adhesion molecules, transmembrane receptors, and ligands. $N$-linked protein glycosylation is essential for the proper subcellular distribution of transmembrane receptors. Glycosylation sites are located in extracellular domains; therefore, the pattern and burden of glycosylation also modulate protein-protein interactions, including receptor activation. GALE-deficient cells display significant cell surface hypoglycosylation with a reduction of gal, galNAc, and sialic acid content in glycoproteins [Broussard et al., 2020]. Of interest, the survival and proliferation of hematopoietic precursors are finely regulated by receptors with tyrosine kinase activity. For example, mild inhibition of $\mathrm{N}$-glycosylation in Fmslike tyrosine kinase 3 (FLT3)-internal tandem duplications-transformed cells is sufficient to misplace and attenuate the activity of FLT3, mediate growth inhibition, 
and induce an endoplasmic reticulum stress response leading to apoptosis [Tsitsipatis et al., 2017]. Hematopoietic cells in culture increase the generation of $\mathrm{N}$-glycans in response to hypoxia, modulating the susceptibility to apoptosis [Pang et al., 2018]. Moreover, null GALE-mutant cells in culture show hypersensitivity to Fas ligandinduced apoptosis due to Fas hypoglycosylation [Broussard et al., 2020]. Knocking down GALE expression in vitro slows the proliferation of megakaryocytic precursors [Seo et al., 2019]. This evidence supports that receptor hypoglycosylation in GALE-deficient cells may decrease pro-survival signals and increase cell death, explaining why hematopoietic precursors undergo apoptosis and abnormal maturation with increased hemophagocytosis in situations of hematologic stress and increased demand such as sepsis.

The patient's congenital heart disease can also be associated with problems in $\mathrm{N}$-glycosylation. From all the phases of heart development, determination of the length, shape, and position of leaflets are finely regulated by paracrine signals. Patterns of cellular migration and accurate chronologic on-off switching of epithelial-to-mesenchymal transition (EMT) pathways are central in heart valve morphogenesis being mediated by $\mathrm{N}$-glycosylated proteins, including protocadherin-16, NOTCH signaling mediators, and ephrin A1 among others [Fischer et al., 2007; Frieden et al., 2010; Durst et al, 2015]. For example, ephrin A1, a ligand for Eph receptor tyrosine kinase, is essential to silence EMT during valve elongation and avoid an excessive accumulation of cells that can lead to redundant leaflets [Frieden et al., 2010]. Deglycosylated ephrin-A1 is unable to activate its receptor [Ferluga et al., 2013]. Some mutations in fibroblastic growth factor receptors that diminish their glycosylation cause malformation of cranium and limbs due to limited cellular sublocalization and autoactivation [Hatch et al., 2006], which may potentially explain why some GALE-deficient individuals develop dysmorphic features.

Pyloric stenosis can occur as an isolated condition or in conjunction with other congenital anomalies in syndromic presentations [Galea and Said, 2018]. Although numerous genetic and environmental factors increase the risk of pyloric stenosis, an association with galactosemia has not been described. Of interest, combined pyloric stenosis and congenital heart defects are identified at increasing rates [Feenstra et al., 2019]. Several genomic variants with dual contributions to normal heart and foregut development have been proposed as a common explanation [Feenstra et al., 2019]. Our GO database exploration revealed $\mathrm{NOTCH} 1$ as a common putative target containing several residues subject to $\mathrm{N}$-glycosylation. However, there are no animal or in vitro models to support a definitive link between NOTCH1 signaling disruption and a pyloric stenosis phenotype. The pathogenesis of pyloric stenosis is likely multifactorial, involving different pathophysiological pathways [Peeters et al., 2012]. At this point, any association between GALE deficiency and pyloric stenosis is conjectural.

Linking GALE deficiency with abnormalities in protein glycosylation opens a new window for treatment opportunities. For example, gal supplementation can restore the biosynthesis of UDP-galNAc and galNAc-bearing glycans in GALE null cells [Broussard et al., 2020]. Therefore, some individuals may benefit from receiving a trace amount of gal, instead of a fully restricted diet [FridovichKeil et al., 2016]. Hematologic abnormalities may not improve after dietary restrictions but can be potentially corrected with bone marrow transplantation [Seo et al., 2019]. For that reason, the patient is currently being followed up by our bone marrow failure and transplant team.

\section{Conclusion}

The phenotype and outcomes of non-generalized forms of GALE deficiency remain poorly understood. Currently, with the wide implementation of comprehensive molecular tests such as WGS, new manifestations are being recognized as part of the GALE deficiency phenotype. Herein, we described a child with the GALE variants p.R51W and p.G237D in association with complex congenital heart disease and bone marrow dysfunction. Although further case studies are needed to determine causality between GALE deficiency and abnormal organ morphogenesis or hematologic disorders, alterations in cell surface protein glycosylation provide a theoretical framework to understand the broad spectrum of GALE deficiency manifestations.

\section{Acknowledgements}

We would like to thank: the Nicklaus Children's Hospital Personalized Medicine Initiative (NCHPMI) Investigators, in alphabetical order: Marilyn Brown, Fabian Castillo, Jennifer McCafferty-Fernandez, and Katherine Schain; the Karius Inc, Redwood City, CA for performing next-generation sequencing for microbial cell-free DNA, and the Mayo Clinic Laboratories, Rochester, $\mathrm{MN}$ for performing GALT and GALE enzymatic activity, gal1-phosphate and urine galactitol tests. We would like to also recognize the contribution of the medical and nursing staff at Nicklaus Children's Hospital in the care of this patient. 


\section{Statement of Ethics}

Appropriate parental consent was obtained according to Nicklaus Children's Hospital policies. Genetic testing protocols applied in this case report have been approved by the Nicklaus Children's Hospital Institutional Review Board (IRB).

\section{Conflict of Interest Statement}

The authors have no conflicts of interest to declare.

\section{Funding Sources}

This study was supported by the Florida Department of Heath State Appropriation, Cornelia T. Bailey Foundation, Sanford Health and Nicklaus Children's Health Care Foundation, Rady
Children's Hospital, and National Institute of Child Health and Human Development and National Human Genome Research Institute grant U19HD077693.

\section{Author Contributions}

C.A.F.A wrote the manuscript and designed the tables and figures with input of all authors. C.A.F.A, L.P., and J.R.G.S. devised the main conceptual ideas. L.P. and C.A.F.A evaluated the bone marrow and peripheral blood histopathology. M.S.W. carried out the techni$\mathrm{cal}$ and analytical aspects of the phenotype-driven next-generation sequencing protocol. C.A.F.A and A.J.F.A performed the computational modeling of GALE structure, evolutionary analysis, and GO database exploration. O.M., J.R.G.S., J.S., and P.J. were the primary care physicians. J.S., P.J., A.J., M.D.B., M.J., B.T., and D.S. (on behalf of the NCHPMI) designed and administrated the comprehensive protocols for targeted diagnostics and discovery of disease association. All authors approved the final version of this manuscript.

\section{References}

Broussard A, Florwick A, Desbiens C, Nischan N, Robertson C, et al. The human UDP-galactose 4'-epimerase (GALE) is required for cell-surface glycome structure and function. J Biol Chem. 2020;295(5):1225-39.

DeClue TJ, Malone JI, Tedesco TA. Florida newborn screening for galactosemia. J Fla Med Assoc. 1991;78(6):369-71.

Dias Costa F, Ferdinandusse S, Pinto C, Dias A, Keldermans L, Quelhas D, et al. Galactose Epimerase Deficiency: Expanding the Phenotype. JIMD Rep. 2017;37:19-25.

Durst R, Sauls K, Peal DS, deVlaming A, Toomer $\mathrm{K}$, Leyne $\mathrm{M}$, et al. Mutations in DCHS1 cause mitral valve prolapse. Nature. 2015;525(7567): 109-13.

Feenstra B, Gørtz S, Lund M, Ranthe MF, Geller F, Melbye M. Co-occurrence of infantile hypertrophic pyloric stenosis and congenital heart defects: a nationwide cohort study. Pediatr Res. 2019;85(7):955-60.

Ferluga S, Hantgan R, Goldgur Y, Himanen JP, Nikolov DB, Debinski W. Biological and structural characterization of glycosylation on ephrin-A1, a preferred ligand for EphA2 receptor tyrosine kinase. J Biol Chem. 2013;288(25): 18448-57.

Fischer A, Steidl C, Wagner TU, Lang E, Jakob PM, Friedl P, et al. Combined loss of Heyl and HeyL causes congenital heart defects because of impaired epithelial to mesenchymal transition. Circ Res. 2007;100(6):856-63.

Fridovich-Keil J, Bean L, He M, Schroer R. Epimerase Deficiency Galactosemia. In: Adam MP, Ardinger HH, Pagon RA, et al., editors. GeneReviews ${ }^{\circledast}$ [Internet]. Seattle (WA): University of Washington, Seattle; 1993-2020.

Frieden LA, Townsend TA, Vaught DB, Delaughter DM, Hwang Y, Barnett JV, et al. Regulation of heart valve morphogenesis by Eph receptor ligand, ephrin-A1. Dev Dyn. 2010;239(12): 3226-34.
Galea R, Said E. Infantile hypertrophic pyloric stenosis: an epidemiological review. Neonatal Netw. 2018;37(4):197-204.

Hatch NE, Hudson M, Seto ML, Cunningham ML, Bothwell M. Intracellular retention, degradation, and signaling of glycosylation-deficient FGFR2 and craniosynostosis syndrome-associated FGFR2C278F. J Biol Chem. 2006; 281(37):27292-305.

Holden HM, Rayment I, Thoden JB. Structure and function of enzymes of the Leloir pathway for galactose metabolism. J Biol Chem. 2003; 278(45):43885-8.

Köhler S, Vasilevsky NA, Engelstad M, Foster E, McMurry J, Aymé S, et al. The Human Phenotype Ontology in 2017. Nucleic Acids Res. 2017;45(D1):D865-76.

McCorvie TJ, Liu Y, Frazer A, Gleason TJ, Fridovich-Keil JL, Timson DJ. Altered cofactor binding affects stability and activity of human UDP-galactose 4'-epimerase: implications for type III galactosemia. Biochim Biophys Acta. 2012;1822(10):1516-26.

Openo KK, Schulz JM, Vargas CA, Orton CS, Epstein MP, Schnur RE, et al. Epimerase-deficiency galactosemia is not a binary condition. Am J Hum Genet. 2006;78(1):89-102.

Pang X, Wang Y, Zhang S, Tan Z, Guo J, Guan F, et al. Altered susceptibility to apoptosis and Nglycan profiles of hematopoietic KGla cells following coculture with bone marrowderived stromal cells under hypoxic conditions. Oncol Rep. 2018;40(3):1477-86.

Peeters B, Benninga MA, Hennekam RC. Infantile hypertrophic pyloric stenosis--genetics and syndromes. Nat Rev Gastroenterol Hepatol. 2012;9(11):646-60.

Pyhtila BM, Shaw KA, Neumann SE, SE, Fridovich-Keil JL. Newborn screening for galactosemia in the United States: looking back, looking around, and looking ahead. JIMD Rep. 2015; 15:79-93.
Richards S, Aziz N, Bale S, Bick D, Das S, GastierFoster J, et al. Standards and guidelines for the interpretation of sequence variants: a joint consensus recommendation of the American College of Medical Genetics and Genomics and the Association for Molecular Pathology. Genet Med. 2015;17(5):405-24.

Schulz JM, Watson AL, Sanders R, Ross KL, Thoden JB, Holden HM, et al. Determinants of function and substrate specificity in human UDP-galactose 4'-epimerase. J Biol Chem. 2004;279(31):32796-803.

Seo A, Gulsuner S, Pierce S, Ben-Harosh M, Shalev $\mathrm{H}$, Walsh T, et al. Inherited thrombocytopenia associated with mutation of UDP-galactose4-epimerase (GALE). Hum Mol Genet. 2019; 28(1):133-42.

Timson DJ, Lindert S. Comparison of dynamics of wildtype and V94M human UDP-galactose 4-epimerase-A computational perspective on severe epimerase-deficiency galactosemia. Gene. 2013;526(2):318-24.

Tsitsipatis D, Jayavelu AK, Müller JP, Bauer R, Schmidt-Arras D, Mahboobi S, et al. Synergistic killing of FLT3ITD-positive AML cells by combined inhibition of tyrosine-kinase activity and N-glycosylation. Oncotarget. 2017; 8(16):26613-24.

Wasilenko J, Lucas ME, Thoden JB, Holden HM, Fridovich-Keil JL. Functional characterization of the K257R and G319E-hGALE alleles found in patients with ostensibly peripheral epimerase deficiency galactosemia. Mol Genet Metab. 2005;84(1):32-8.

Wohlers TM, Christacos NC, Harreman MT, Fridovich-Keil JL. Identification and characterization of a mutation, in the human UDP-galactose-4-epimerase gene, associated with generalized epimerase-deficiency galactosemia. Am J Hum Genet. 1999;64(2):462-70.

Yang H, Robinson PN, Wang K. Phenolyzer: phenotype-based prioritization of candidate genes for human diseases. Nat Methods. 2015;12(9): 841-3.
GALE Deficiency Associated with Dyshematopoiesis and Congenital Heart Disease 